\title{
Effect of Retinal Injury Induced by Laser Photocoagulation on Visuospatial Memory in Mouse Model
}

\author{
Priya Mehra ${ }^{1,2}$ Parul Bali ${ }^{3}$ Jagtar Singh ${ }^{1}$ Pradip Kumar Saha ${ }^{4}$ Akshay Anand ${ }^{2}$
}

${ }^{1}$ Department of Biotechnology, Panjab University, Chandigarh, India

${ }^{2}$ Neuroscience Research Lab, Department of Neurology, Postgraduate Institute of Medical Education and Research, Chandigarh, India

${ }^{3}$ Department of Biological Science, IISER - Indian Institute of Science Education and Research, Mohali, India

${ }^{4}$ Department of Obstetrics and Gynecology, Postgraduate Institute of Medical Education and Research, Chandigarh, India

\author{
Address for correspondence Parul Bali, PhD, Department of \\ Biological Science, IISER - Indian Institute of Science Education and \\ Research, Mohali 140306, Punjab, India \\ (e-mail: parul28bali@gmail.com); \\ Akshay Anand, PhD, Neuroscience Research Lab, Department \\ of Neurology, Postgraduate Institute of Medical Education and \\ Research, Chandigarh 160012, India \\ (e-mail: akshay1anand@rediffmail.com).
}

\author{
Abstract \\ Keywords \\ - visual impairment \\ - laser photocoagulation \\ - retinal degeneration \\ - cognition \\ - memory
}

Visual pathway reveals the connection between neuronal activity of the brain and eye. The neural networks of brain amplify the retinal signals resulting in the formation of visual image. The laser injury in the retina may affect the visual pathway and may lead to disruption of neuronal signals/activity. Therefore, we aimed to study the effect of retinal injury induced by laser on cognitive abilities in laser-induced mouse model. We have established laser model to understand the relation between retina and brain by disrupting retinal pigment epithelial (RPE) layer and evaluate the effect of laser-induced retinal injury on visuospatial memory. Age- and sex-matched C57BL/6] male mice were taken for conducting the experiments. The laser model was established by using laser photocoagulator to disrupt the RPE layer of the retina. After defined irradiation of laser onto mouse retina, the fundus fluorescein angiography was performed to confirm the laser spots. The visuospatial and short-term memory was performed using neurobehavioral test, that is, Morris water maze (MWM), and passive avoidance, respectively. In MWM experiment, results showed that escape latency time, which was taken by healthy and laser-injured mice was comparable. This was further validated by another neurobehavioral analysis, that is, passive avoidance that showed nonsignificant difference between these two groups using independent $t$-test. Visuospatial memory may not be affected by retinal injury induced by laser photocoagulation. It may depend on the power of the laser and duration of the laser. The severe injury in the retina such as optic nerve damage may cause dysfunctioning of visual pathway. published online June 25, 2021
DOI https://doi.org/

$10.1055 / \mathrm{s}-0041-1730747$ ISSN 0976-3147 (c) 2021. Association for Helping Neurosurgical Sick People.

This is an open access article published by Thieme under the terms of the Creative Commons Attribution-NonDerivative-NonCommercial-License, permitting copying and reproduction so long as the original work is given appropriate credit. Contents may not be used for commercial purposes, or adapted, remixed, transformed or built upon. (https://creativecommons.org/licenses/by-nc-nd/4.0/).

Thieme Medical and Scientific Publishers Pvt. Ltd. A-12, 2nd Floor, Sector 2, Noida-201301 UP, India 


\section{Introduction}

Several eye disorders such as age-related macular degeneration (AMD), diabetic retinopathy, glaucoma, retinal vein occlusion, retinal ischemia, and retinal pigmentosa have retinal degeneration as a main symptom. It is considered as one of the major causes of blindness.

Visual impairment affects retina, optic nerve, and different parts of brain such as thalamus, optic chiasma, optic radiation, occipital lobes, and visual cortex. According to the World Health Organization, globally 285 million people are visual impaired, out of which 39 million are blind and majority of people are above 50 years of age or more, ${ }^{1}$ whereas 123.7 million people are with refractive errors, 6.9 million with glaucoma, 65.2 million with cataract, 3 million with diabetic retinopathy, and 11 million with AMD, and it is expected to double in next 10 years. ${ }^{2}$ In developing countries, leading causes of visual impairment are AMD, glaucoma, and diabetic retinopathy.

However, the prevalence of visual impairment is expected to increase twofold by 2050.,4 Moreover, there is a close relationship between visual impairment and cognitive decline as both are neurodegenerative disorders and are related to aging. ${ }^{5,6}$ The study showed the relation between macular degeneration and Alzheimer's disease (AD) in older age group resulting in poor reading ability, distorted blurred faces due to lack of sensory stimulus, and brain stimulus activity. ${ }^{7}$ It was studied that visual performance has significant effect on memory deterioration. ${ }^{8}$ A study reported correlation of memory and learning with vision in 1,000 cataract-operated patients and found significant improvement in memory task and learning. ${ }^{9}$ Therefore, such results show that memory and learning are significantly associated with vision.

Various research have shown the association of visual performance with cognition function..$^{6,7}$ The dysregulations of blood retinal barriers has implicated age-related cognitive decline. ${ }^{10}$ In neurological disorders, disruption of retinal blood barrier leads to cognitive decline as signaling get disrupted..$^{11}$ Laser model has been used to study the visuospatial memory by either disrupting retinal layers or optic nerve that transmits signal from retina to brain where visual image formation occur known as visual perception. ${ }^{12}$ The primary site of injury using laser photocoagulation is retina. There are two methods by which retina get disrupted by laser, that is, one by targeting the Bruch's membrane that is an innermost layer of the choroid below retinal pigment epithelial (RPE) layer. The proliferation of choroidal vessels takes place that penetrate RPE through Bruch's membrane and leakage of lipids and blood from these new fragile blood vessels under the retina and sometimes between the retinal layers also occurs. This process is known as choroidal neovascularization. ${ }^{13}$ Second method is by directly targeting the RPE that absorb laser beam, which gets converted into thermal energy leading to denaturation of cellular proteins. ${ }^{14}$ The retinal injury model can be validated by visualizing damaged retina through fundus fluorescein angiography (FFA), optical coherence tomography, and electroretinogram. In our study, we have established the laser model and tried to investigate that whether the retinal injury affects visuospatial memory.

Some studies have shown that visual impairment leads to visual cortex damage. It has shown that neuronal cells in the retina as well as its thickness also get affected in AD. ${ }^{15,16}$ The retina shares various functions with central nervous system, transmission of signal leads to visual perception, cognition, image formation, and spatial memory. Therefore, keeping in mind this doubted or unconfirmed relation between visual problems and visuospatial memory or cognitive learning, this laser-induced retinal injury model was established. This model was used to study the effects of laser on visuospatial memory or cognitive abilities via retinal degeneration using standardized parameters such as $100-\mu \mathrm{m}$ size of the laser.

\section{Methods}

All the experiments were performed under good laboratory practice guidelines. All the protocols were approved by the study director and quality assurance personnel.

\section{Study Design}

In this study, 6 to 8 weeks C57BL/6J male mice were used. Animals were fed on standard diet and RO drinking water was available ad libitum. Animals were maintained in a 12-hour light/dark cycle. Utmost care was taken while performing experiments. All the experiments were conducted in accordance with Institutional Animal Ethical Committee (105/IAEC/720).

\section{Laser Photocoagulation}

Mice were anesthetized with the mixture of ketamine and xylazine in the ratio 1:10 intraperitoneally in to mice and their pupil was dilated with $1 \%$ tropicamide and phenylephrine. A slit lamp connected with laser photocoagulator (IRIDEX) with $532 \mathrm{~nm}$ argon green laser wavelength was used. Eight laser shots were given to both eyes. The laser shots were given around optic disc in each eye with coverslips as a contact lens. The size of laser spots was $100 \mu \mathrm{m}$; duration of exposure to laser pulse was 100 milliseconds; power of the laser was $200 \mathrm{~mW}$. These all parameters were already standardized in our laboratory. ${ }^{17}$ The mice whiskers were trimmed and eyes were kept moist and clean with sterile saline. After that, mice were placed on slit lamp laser system. The fundus was observed using coverslip with one drop of hydroxypropyl methylcellulose. Once the site got visualized, eight laser shots were given to each eye in circular fashion around the optic disc.

\section{Fundus Fluorescein Angiography}

To validate the laser model, FFA was done after 24 hours of laser injury. The animals were anesthetized and pupil was dilated. The mice received tail vein injection of $0.2 \mu \mathrm{L}$ of fluorescein dye diluted in $1 \mathrm{~mL}$ normal saline. Immediately 
after injecting, fundus was focused. The vascular leakage and size of laser lesion was determined by using Heidelberg Engineering software. The laser-induced leakage validated the establishment of laser model.

\section{Neurobehavioral Analysis}

\section{Rotarod}

The rotarod consists of circular rod rotating at constant speed to measure muscular strength, motor coordination of mice. After 1 month of laser injury, neurobehavioral assessment was done. The mice were kept on rotating rod at constant speed of $15 \mathrm{rpm}$ for 180 seconds. The mice were allowed to rotate on rotarod for 3 minutes to maintain the balance and to avoid falling. The time taken by the mice to fall from rotating rod was recorded. The trial was done for three times daily followed by Morris water maze (MWM).

\section{Morris Water Maze}

MWM was performed to measure the spatial memory of the mice. After laser injury, the mice underwent neurobehavioral analysis. It was 7 days protocol, first 6 days were acquisition days and the seventh day was retrieval day. Before MWM, mice were screened for swimming ability by rotarod experimentation. MWM consists of circular tank filled with water with equally divided four quadrants named Q1, Q2, Q3, and Q4. A circular white platform was submerged $1 \mathrm{~cm}$ below the water surface kept in Q1 quadrant. The visual cues were placed on the walls and pool side. The background of water was colored white to identify and track the darker mice against bright background in ANY-maze software. The complete protocol was recorded and tracked by using ANY-maze video software connected with webcam. The water temperature was maintained between 21 and $24^{\circ} \mathrm{C}$. Four trials were done every day and each trial was for 120 seconds. ANYmaze software measures various parameters such as escape latency time of the mice, mean speed from platform to different quadrant, time spent by mice in each quadrant, distance traveled by mice from each quadrant to platform, and mean distance from the platform.

\section{Passive Avoidance}

Passive avoidance is the learning task used to measure short-term memory. The instrument divided into two equal parts named light chamber and dark chamber with a gate between two. This protocol was performed for 3 days for 10 minutes. On first day, mice were allowed to explore both chambers freely. On second day (acquisition day) mice were kept in the light chamber and gate was opened after 30 seconds and then time was recorded when mice entered the dark chamber. In dark chamber, the mice received mild foot shock of $20 \mathrm{~mW}$ and after that mice were removed immediately from the box. On third day (test day), the time spent by mice in light chamber was recorded to avoid foot shock in dark.

\section{Enucleation of Eye}

Mice were euthanized by overdose of ketamine: xylazine combination. The eyes were enucleated and stored at $80^{\circ} \mathrm{C}$ for further experiments.

\section{Results}

\section{Retinal Assessment}

FFA: The fundus imaging was done after 24 hours of laser injury. The vascular leakage at the injury site showing laser lesions in eight laser group. The images were captured by Heidelberg Engineering software as shown in - Fig. 1.
A. Healthy Group
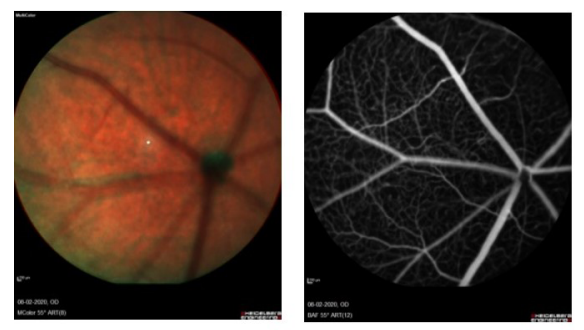

Right Eye

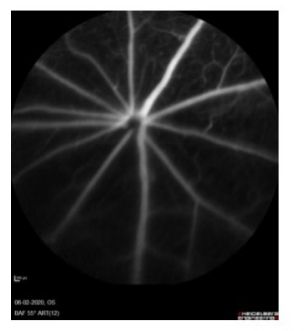

Left Eye

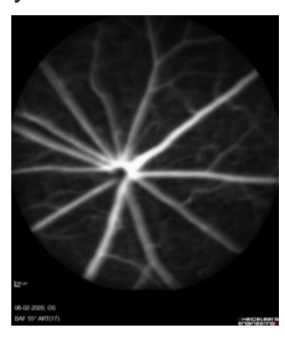

Fig. 1 Fundus fluorescein angiography shown fundus image of the eye. (A) The healthy control group shows no leakage of fluorescein dye in left and right eyes. (B) Although in eight laser group, the white arrows show leakage at injury site in both right and left eyes of laser group after 24 hours of laser injury. 

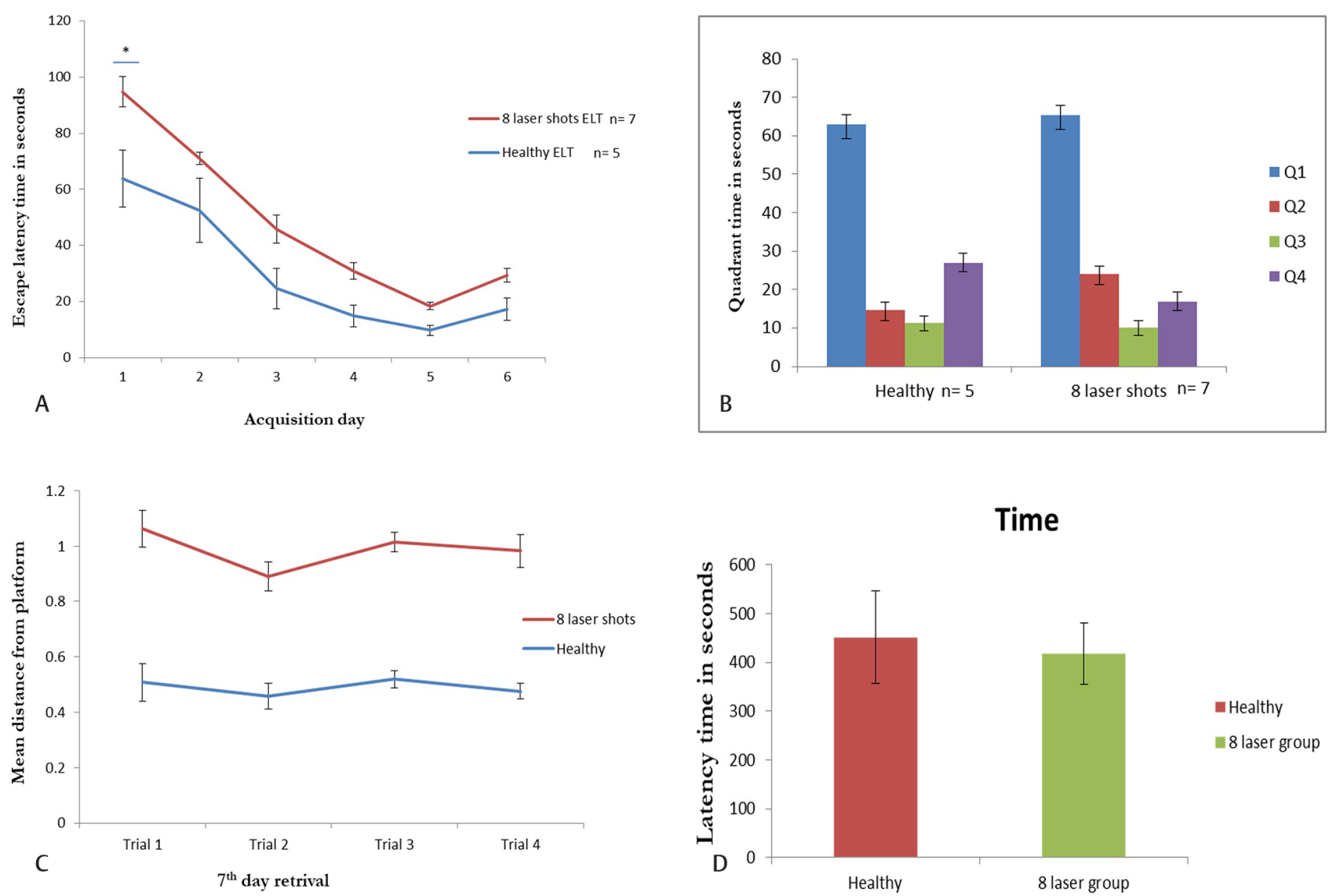

Fig. 2 The MWM analysis showing learning in mice. (A) The day-wise escape latency time during acquisition days of mice in healthy control $(N=5)$ and eight laser injury $(N=7)$. The ELT was found to be more in mice with eight laser group at day 1 as compared with the healthy control group. (B) Quadrant time: The time spent by mice in MWM quadrants Q1, Q2, Q3, and Q4 on retrieval day (seventh day). (C) The search error measured as mean distance from hidden platform traveled was less in healthy group as compared with laser group but nonsignificant. (D) Passive avoidance found more latency in eight laser group as compared with healthy group. The trial lasts for maximum 10 minutes. ELT, escape latency time; MWM, Morris water maze.

\section{Neurobehavioral Assessment}

Morris water maze: Before MWM, the mice were undergone rotarod experiment to determine the swimming ability. All mice were found to be swim well, and their motor coordination and muscular strength was strong. After three trials of 3 minutes, mice had undergone MWM experiment. ANY-maze software was attached to webcam to track the mice. It was found significant reduction in the swimming path on day 1 in healthy group as compared with laser group ( - Fig. 2A). However, all mice were learned to reach the platform but were nonsignificantly. - Fig. 2B depicts that the mice spent time mice in each quadrant at seventh day of the experiment. It was found that mice spent more time in Q1 quadrant where the hidden platform was placed but nonsignificantly, whereas, mice spent maximum time in Q2 and Q4 quadrants significantly ( - Fig. 2B). The mean distance from platform was found to be nonsignificant in both groups ( - Fig. $2 \mathbf{C}$ ).

\section{Passive Avoidance}

After MWM, to confirm the learning and memory ability, the mice were subjected to passive avoidance in which time spent by mice in the light chamber to avoid shock was noted. We found that mice had learned and remain in the light chamber maximum time for 10 minutes on day 3 in both

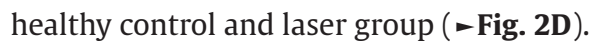

\section{Statistical Analysis}

IBM SPSS version 22 was used for the statistical analysis, whereas baseline values were checked for normalcy and baseline group comparisons were done by using independent samples $t$-test.

\section{Discussion}

Visual system is the process in which signals get transmitted, processed, and interpreted by axonal retinal ganglion cells and primary cortex in brain to form visual perception. The neuronal cells in the retina extend axons from retinal ganglion cells to optic nerve to brain mainly in primary cortex to form image. Brain is able to remember entire series of images formed by retina when we move eye results in spatial memory. Since the image produced by eye is directly correlated with the brain ${ }^{18}$ as the electrical signal get transmitted from retinal photoreceptors cells to brain through optic nerve which is responsible for visual perception, thus impairment 
in the visual function will affect the brain function and understanding directly. Understanding the mechanism of visual and cognitive function will provide evidence to understand the disease with another important perspective. This invokes the need to study the effects of visual impairment on brain function and to study the effect of the treatment used for vision repair on the brain function.

In retinal degenerations such as retinitis pigmentosa and AMD, diabetic retinopathy begins with loss of photoreceptor, impaired macula and later on results in loss of inner retinal neurons, but significant numbers of bipolar and ganglion cells remain for many years that can be reversed by electrical stimulation of the retina or the optic nerve. ${ }^{19}$

In our study, we observed that laser-induced retinal injury do not affects the learning and memory. In spatial memory test, laser-injured mice showed learning and memorizing at the retrieval day when the hidden platform was removed. This test was validated by fear-motivated test in which laser-injured as well as healthy mice spent maximum time in light chamber to avoid foot shock. Some of the studies have shown that learning and visual memory may not be correlated. Yancopoulos (2016) used anti-VEGF treatment to abolish clinical neovascularization; however, investigations to examine its association with cognitive improvement have been lacking. While they found significant results in the treatment of angiogenic eye disorders, they did not report the impact on neurobehavioral. ${ }^{13,20}$ Prusky et al examined the role of visual acuity in animal model and found that the mice with reduced visual acuity were found to be deficit in place of learning and do not differ their learning ability in MWM. ${ }^{21}$ Similarly, Storchi et al demonstrated that innate behavior has various advantages in vision test mainly by determining the visual capabilities with intact visual function in mice model and in retinal degeneration..$^{22}$

Hence, we studied the association between laser-induced retinal injury and memory in mice model. Many studies have shown that the neuronal and retinal degeneration are correlated with each other. Neuronal cell death results in cognitive and visual impairment. Also, neurodegeneration affects retina. In neurological disorders such as $A D$ and Parkinson's disease, retinal thickness reduced in mild cognition impairment as compared with healthy control group. ${ }^{23,24}$ Although various studies from our group have independently analyzed the role of risk and genetic factors in onset of neurodegeneration, we had not examined the association between two clinical situations, that is, vision loss and cognitive deficit. ${ }^{25-43}$ However, there are studies that have shown the correlation between visual impairment and cognition in the population and found significant association between them. ${ }^{44-46}$

Oktem et al found significant correlation between retinal thickness and mild cognition in AD patients as compared with control group ${ }^{47}$ However, our results have shown that visuospatial memory is independent of retinal injury induced by laser photocoagulation.

\section{Limitations of the Study}

The molecular analysis such as reverse transcription polymerase chain reaction, immunohistochemical staining to check the expression of different neuronal marker and apoptotic marker is yet to be done. The samples were stored at $-80^{\circ} \mathrm{C}$ for further experimentation.

\section{Conclusion}

Visuospatial memory may not affect by retinal injury induced by laser photocoagulation. It may depend on the power of the laser and duration of the laser. The severe injury in the retina such as optic nerve damage may cause dysfunctioning of visual pathway.

\section{Ethical Approval}

The ethical approval was taken by Institute Animal Ethical committee. The ethical approval number is 105/IAEC/720.

\section{Funding}

None.

\section{Conflict of Interest}

None declared.

\section{Acknowledgment}

The authors acknowledge Mr. Arun Kapil from the Department of Opthalmology, PGIMER, for Fundus Fluorescein Fngiography FFA imaging.

\section{References}

1 World Health Organization, Blindness and Vision Impairment Prevention. World Health Organization; 2020. Available at: https://www.who.int/news-room/fact-sheets/detail/blindnessand-visual-impairment

2 World Health Organization, Blindness and Vision Impairment. World Health Organization; 2020. Available at: https://www. who.int/blindness/Vision2020_report.pdf

3 Flaxman SR, Bourne RR, Resnikoff S, et al. Vision Loss Expert Group of the Global Burden of Disease Study. Global causes of blindness and distance vision impairment 1990-2020: a systematic review and meta-analysis. Lancet Glob Health 2017;5(12):e1221-e1234

4 Stevens GA, White RA, Flaxman SR, et al. Vision Loss Expert Group. Global prevalence of vision impairment and blindness: magnitude and temporal trends, 1990-2010. Ophthalmology 2013;120(12):2377-2384

5 Uhlmann RF, Larson EB, Koepsell TD, Rees TS, Duckert LG. Visual impairment and cognitive dysfunction in Alzheimer's disease. J Gen Intern Med 1991;6(2):126-132

6 Salthouse TA, Hancock HE, Meinz EJ, Hambrick DZ. Interrelations of age, visual acuity, and cognitive functioning. J Gerontol B Psychol Sci Soc Sci 1996;51(6):317-330

7 Klaver CC, Ott A, Hofman A, AssinkJJ, Breteler MM, de Jong PT. Is age-related maculopathy associated with Alzheimer's disease? The Rotterdam study. Am J Epidemiol 1999;150(9):963-968

8 Anstey KJ, Luszcz MA, Sanchez L. Two-year decline in vision but not hearing is associated with memory decline in very old adults in a population-based sample. Gerontology 2001;47(5):289-293 
9 Fagerström R. Correlations of memory and learning with vision in aged patients before and after a cataract operation. Psychol Rep 1992;71(3 Pt 1):675-686

10 Park MH, Lee JY, Park KH, et al. Vascular and neurogenic rejuvenation in aging mice by modulation of ASM. Neuron 2018;100(1):167-182.e9

11 Venkat P, Chopp M, Chen J. Blood-brain barrier disruption, vascular impairment, and ischemia/reperfusion damage in diabetic stroke. J Am Heart Assoc 2017;6(6):e005819

12 Birtel J, Harmening WM, Krohne TU. Holz FG, Charbel Issa P, Herrmann P. Retinal injury following laser pointer exposure: a systematic review and case series. Dtsch Arztebl Int 2017;114(49):831-837

13 Ambati J, Fowler BJ. Mechanisms of age-related macular degeneration. Neuron 2012;75(1):26-39

14 Glickman RD. Phototoxicity to the retina: mechanisms of damage. Int J Toxicol 2002;21(6):473-490

15 Armstrong RA. Visual field defects in Alzheimer's disease patients may reflect differential pathology in the primary visual cortex. Optom Vis Sci 1996;73(11):677-682

16 Parisi V, Restuccia R, Fattapposta F, Mina C, Bucci MG, Pierelli F. Morphological and functional retinal impairment in Alzheimer's disease patients. Clin Neurophysiol 2001;112(10):1860-1867

17 Bammidi S, Bali P, Kalra J, Anand A. Transplantation efficacy of human ciliary epithelium cells from fetal eye and Lin -ve stem cells from umbilical cord blood in the murine retinal degeneration model of laser injury. Cell Transplant 2020;29:963689720946031

18 Quigley HA, Sanchez RM, Dunkelberger GR, L'Hernault NL, Baginski TA. Chronic glaucoma selectively damages large optic nerve fibers. Invest Ophthalmol Vis Sci 1987 28(6):913-920

19 Loewenstein JI, Montezuma SR, Rizzo JF II. Outer retinal degeneration: an electronic retinal prosthesis as a treatment strategy. Arch Ophthalmol 2004;122(4):587-596

20 Yancopoulos GD. Use of a VEGF antagonist to treat angiogenic eye disorders. Google Patents. 2016

21 Prusky GT, West PW, Douglas RM. Reduced visual acuity impairs place but not cued learning in the Morris water task. Behav Brain Res 2000;116(2):135-140

22 Storchi R, Rodgers J, Gracey M, et al. Measuring vision using innate behaviours in mice with intact and impaired retina function. Sci Rep 2019 9(1):10396

23 Akar G, Gozke E, Agirman Y, Kurna SA. Retinal nerve fiber layer thickness in cases with mild cognitive impairment and Alzheimer-type dementia. Biomed Res 2014;25(4):597-602

24 Oktem EO, Derle E, Kibaroglu S, Oktem C, Akkoyun I, Can U. The relationship between the degree of cognitive impairment and retinal nerve fiber layer thickness. Neurol Sci 2015;36(7):1141-1146

25 Anand A, Banik A, Thakur K, Masters CL. The animal models of dementia and Alzheimer's disease for pre-clinical testing and clinical translation. Curr Alzheimer Res 2012;9(9):1010-1029

26 Anand A, Gupta PK, Sharma NK, Prabhakar S. Soluble VEGFR1 (sVEGFR1) as a novel marker of amyotrophic lateral sclerosis (ALS) in the North Indian ALS patients. Eur J Neurol 2012;19(5):788-792

27 Anand A, Saraf MK, Prabhakar S. Sustained inhibition of brotizolam induced anterograde amnesia by norharmane and retrograde amnesia by L-glutamic acid in mice. Behav Brain Res 2007;182(1):12-20

28 Anand A, Saraf MK, Prabhakar S. Antiamnesic effect of B. monniera on L-NNA induced amnesia involves calmodulin. Neurochem Res 2010;35(8):1172-1181
29 Anand A, Sharma NK, Gupta A, et al. Single nucleotide polymorphisms in $\mathrm{MCP}-1$ and its receptor are associated with the risk of age related macular degeneration. PLoS One 2012;7(11):e49905

30 Anand A, Thakur K, Gupta PK. ALS and oxidative stress: the neurovascular scenario. Oxid Med Cell Longev 2013;2013:635831

31 Anand A, Tyagi R, Mohanty M, Goyal M, Silva KR, Wijekoon N. Dystrophin induced cognitive impairment: mechanisms, models and therapeutic strategies. Ann Neurosci 2015;22(2):108-118

32 Banik A, Brown RE, Bamburg J, et al. Translation of pre-clinical studies into successful clinical trials for Alzheimer's disease: what are the roadblocks and how can they be overcome? J Alzheimers Dis 2015;47(4):815-843

33 English D, Sharma NK, Sharma K, Anand A. Neural stem cellstrends and advances. J Cell Biochem 2013;114(4):764-772

34 Goyal K, Koul V, Singh Y, Anand A. Targeted drug delivery to central nervous system (CNS) for the treatment of neurodegenerative disorders: trends and advances. Cent Nerv Syst Agents Med Chem 2014;14(1):43-59

35 Gupta PK, Prabhakar S, Abburi C, Sharma NK, Anand A. Vascular endothelial growth factor-A and chemokine ligand (CCL2) genes are upregulated in peripheral blood mononuclear cells in Indian amyotrophic lateral sclerosis patients. J Neuroinflammation 2011;8(1):114

36 Sharma NK, Gupta A, Prabhakar S, Singh R, Sharma S, Anand A. Single nucleotide polymorphism and serum levels of VEGFR2 are associated with age related macular degeneration. Curr Neurovasc Res 2012;9(4):256-265

37 Mathur D, Goyal K, Koul V, Anand A. The molecular links of re-emerging therapy: a review of evidence of Brahmi (Bacopa monniera) Front Pharmacol 2016;7:44

38 Sharma K, Sharma NK, Anand A. Why AMD is a disease of ageing and not of development: mechanisms and insights. Front Aging Neurosci 2014;6:151

39 Sharma NK, Gupta A, Prabhakar S, Singh R, Bhatt AK, Anand A. CC chemokine receptor-3 as new target for age-related macular degeneration. Gene 2013;523(1):106-111

40 Sharma NK, Gupta A, Prabhakar S, et al. Association between CFH Y $402 \mathrm{H}$ polymorphism and age related macular degeneration in North Indian cohort. PLoS One 2013;8(7):e70193

41 Sharma NK, Prabhakar S, Gupta A, et al. New biomarker for neovascular age-related macular degeneration: eotaxin- 2 . DNA Cell Biol 2012;31(11):1618-1627

42 Singh T, Prabhakar S, Gupta A, Anand A. Recruitment of stem cells into the injured retina after laser injury. Stem Cells Dev 2012;21(3):448-454

43 Vinish M, Prabhakar S, Khullar M, Verma I, Anand A. Genetic screening reveals high frequency of PARK2 mutations and reduced Parkin expression conferring risk for Parkinsonism in North West India. J Neurol Neurosurg Psychiatry 2010;81(2):166-170

44 Spierer O, Fischer N, Barak A, Belkin M. Correlation between vision and cognitive function in the elderly: a cross-sectional study. Medicine (Baltimore) 2016;95(3):e2423

45 Lim ZW, Chee ML, Soh ZD, et al. Association between visual impairment and decline in cognitive function in a multiethnic Asian population. JAMA Netw Open 2020;3(4):e203560

46 de Kok DS, Teh RO, Pillai A, et al. What is the relationship between visual impairment and cognitive function in octogenarians? N Z Med J 2017;130(1460) :33-47

47 Pena MCS, Sobreira EST, Souza CP, Oliveira GN, Tumas V, do Vale FAC. Visuospatial cognitive tests for the evaluation of patients with Parkinson's disease. Dement Neuropsychol 2008;2(3):201-205 